\title{
Rehabilitation Exergames: Use of Motion Sensing and Machine Learning to Quantify Exercise Performance in Healthy Volunteers
}

Reza Haghighi Osgouei ${ }^{1}$, PhD; David Soulsby ${ }^{2}$, BSc; Fernando Bello ${ }^{1}, \mathrm{PhD}$

${ }_{1}^{1}$ Imperial College Centre for Engagement and Simulation Science (ICCESS), Faculty of Medicine, Department of Surgery and Cancer, Imperial College London, London, United Kingdom

${ }^{2}$ Children's Services and Dietetics, Chelsea and Westminster Hospital, London, United Kingdom

\section{Corresponding Author:}

Reza Haghighi Osgouei, PhD

Imperial College Centre for Engagement and Simulation Science (ICCESS)

Faculty of Medicine, Department of Surgery and Cancer

Imperial College London

Room H 3 8, ICCESS, Chelsea and Westminster

369 Fulham Road

London, SW10 9NH

United Kingdom

Phone: 44940419331

Email: r.haghighi-osgouei@imperial.ac.uk

\section{Abstract}

Background: Performing physiotherapy exercises in front of a physiotherapist yields qualitative assessment notes and immediate feedback. However, practicing the exercises at home lacks feedback on how well patients are performing the prescribed tasks. The absence of proper feedback might result in patients performing the exercises incorrectly, which could worsen their condition. We present an approach to generate performance scores to enable tracking the progress by both the patient at home and the physiotherapist in the clinic.

Objective: This study aims to propose the use of 2 machine learning algorithms, dynamic time warping (DTW) and hidden Markov model (HMM), to quantitatively assess the patient's performance with respect to a reference.

Methods: Movement data were recorded using a motion sensor (Kinect V2), capable of detecting 25 joints in the human skeleton model, and were compared with those of a reference. A total of 16 participants were recruited to perform 4 different exercises: shoulder abduction, hip abduction, lunge, and sit-to-stand exercises. Their performance was compared with that of a physiotherapist as a reference.

Results: Both algorithms showed a similar trend in assessing participant performance. However, their sensitivity levels were different. Although DTW was more sensitive to small changes, HMM captured a general view of the performance, being less sensitive to the details.

Conclusions: The chosen algorithms demonstrated their capacity to objectively assess the performance of physical therapy. HMM may be more suitable in the early stages of a physiotherapy program to capture and report general performance, whereas DTW could be used later to focus on the details. The scores enable the patient to monitor their daily performance. They can also be reported back to the physiotherapist to track and assess patient progress, provide feedback, and adjust the exercise program if needed.

(JMIR Rehabil Assist Technol 2020;7(2):e17289) doi: $10.2196 / 17289$

\section{KEYWORDS}

rehabilitation exergames; performance assessment; similarity score; motion sensing; machine learning; dynamic time warping; hidden Markov model 


\section{Introduction}

\section{Background}

Rehabilitation is essential to regain lost or weakened functionality after injury or surgery. Although it is commonly initiated in a clinic and supervised by a physiotherapist, the prescribed therapeutic exercises will normally need to be practiced at home by patients on their own. Lack of motivation and compliance may hinder the healing process and, in some cases, even worsen the injury. Advances in virtual reality technologies have resulted in various virtual rehabilitation platforms introduced to address this issue [1-3]. They are generally equipped with sensory devices to track and monitor the patient's movement when performing the exercises. Among them are systems based on the concept of exergaming and exercise gaming. These are interactive video games with some simple scenarios that enable a patient to perform a therapeutic exercise by playing the game. Exergames are a subgenre of serious games developed for the purpose of encouraging exercise and activity. Although sharing some aspects with medical simulation, in exergames, the emphasis is more on the added educational value of fun and entertainment. Accessibility (not needing the constant presence of a physician) and entertainability (turning repetitive tasks to playful activities) are 2 key advantages of such systems.

The impact of serious games on physical therapy has been studied in terms of effectiveness [4-6] and motivational determinants $[7,8]$. However, as a virtual guide replacing or complementing a real physician, exergaming systems tend to lack objective, clinically meaningful evaluation of patient performance. At best, game scores, the extent to which the player achieved the goals of the game, are reported at the end of the session along with statistics such as completion time. The work presented in this study aims to fill this gap by introducing an approach to compare a patient's performance with that of a reference, using the Medical Interactive Recovery Assistant (MIRA) rehabilitation platform. We explore 2 different machine learning techniques: dynamic time warping (DTW) and hidden Markov model (HMM). They have been widely used for gesture recognition to study acquired motion trajectories [9-14]. The former belongs to model-less time domain methods, whereas the latter is a model-based probabilistic technique for time-series analysis. Comparing the patient's trajectory with that of a physiotherapist as a reference, each approach generates an objective similarity score indicating how similar the performance was.

\section{Related Work}

\section{Rehabilitation Platforms}

The concept of exergaming enables exercising when playing games. For players, it is an opportunity to play games in a more active and less passive manner. For patients, it offers the opportunity to practice therapeutic tasks in a more playful and less repetitive manner. Exergames offer various activities, such as aerobic exercises and dancing; balance and stretching workouts; and recreational simulations, such as golf, skiing, and more. However, they require additional hardware and software. In terms of hardware, they require proper sensory equipment to track the user's motion. In terms of software, the game scenario must accommodate whole body interaction. There are various commercially available game consoles that enable exergames, including Xbox (Microsoft), PlayStation (Sony), and Wii (Nintendo). Each comes with its own dedicated input device for enabling user interaction with the games, that is, Kinect for Xbox, Move for PlayStation, and Remote Plus for Wii.

Among them, Kinect has gained higher popularity owing to its acceptable performance and versatility [15]. Microsoft introduced Kinect in 2010 as a peripheral input device for its gaming console Xbox and discontinued it in 2017. Kinect enables interaction with virtual environments using gestures rather than conventional controllers. The device includes an RGB camera and a depth sensor, which combines full body 3-dimenonal (3D) motion capture capabilities and gesture recognition. Using the Kinect SDK 2.0, the position of 25 human skeleton joints can be accessed with a sample rate of $30 \mathrm{fps}$. Since its launch, researchers have used the Kinect for various applications, including rehabilitation [16-21]. Other similar motion-sensing devices capable of tracking the 3D position of the joints include Azure Kinect (Microsoft), Astra (Orbbec), RealSense (Intel), Structure Sensor (Occipital), and BlasterX Senz3D (Creative).

Kinerehab was introduced by Chang et al [16] to assist therapists in rehabilitating students at a public school. By conducting a user study with two patients, the authors compared two experimental phases, baseline (without assistive technology) and intervention (with Kinerehab), lifting both arms to the front, to the side, and upward. The data showed a significant increase in motivation (willingness to keep practicing) among participants and hence improved exercise performance using Kinerehab compared with performance in the presence of a therapist. A Kinect-based serious game for physiotherapy (KSGphysio) was proposed by Duarte et al [17] that had a mobile interface to facilitate the analysis of patient progress by generating relevant statistics. Cary et al [18] developed a web-based serious game called Therasoup to improve the patient's motivation when performing exercises and to provide technical data to the physiotherapist, which helped in the assessment. The game tries to simulate daily life activities such as cooking, where the player controls an avatar to pick the ingredients from shelves and put them in a pan at the center. Su et al [19] developed a Kinect-enabled home-based rehabilitation system (KEHR) to assist patients in conducting safe and effective off-hospital rehabilitation without the immediate supervision of a physician. KEHR supported 3 different shoulder rehabilitation exercises: shoulder abduction, shoulder anterior elevation, and shoulder external and internal rotation. A serious game framework for therapy (Theragame) providing options to imitate the actions performed by an avatar or to play a game that trains specific parts of the body was introduced by Ferreira et al [20]. A web-based platform for physical telerehabilitation for patients after hip replacement surgery was described by Rybarczyk et al [21] with 2 goals in mind: making use of a low-cost motion capture device (Kinect) and real-time automatic assessment of performance. A comprehensive review of the technical and 
clinical impacts of the Kinect in physical therapy and rehabilitation is given by Mousavi et al [15]. The survey covers rehabilitation systems before and after Kinect as well as platforms with and without clinical evaluation. Although Kinect-based rehabilitation systems are accepted by both patients and therapists, the lack of objective, clinically meaningful evaluation of performance raises questions regarding their effectiveness.

Commercially available rehabilitation platforms based on Kinect and exergames include MIRA [1], VirtualRehab [2], and REHABILITY [3]. All these platforms have the capacity to be used at home to encourage and monitor performance or at a hospital to assist and support physiotherapists. MIRA is a class I medical device that uses games built based on best clinical practice and expertise from specialist physiotherapists to keep patients engaged and motivated throughout the therapy. VirtualRehab, a Conformitè Europëenne-certified class I medical device, is a product that can be used in clinics and hospitals as well as in patients' homes, allowing them to continue their rehabilitation treatment. With REHABILITY, patients can perform rehabilitation exercises at the clinic and hospital or remotely, but with constant medical supervision. None of these systems offers automatic, objective assessment comparing patient performance with that of a reference. They offer game scores such as number of hits and statistics such as execution/completion time.

\section{Performance Evaluation}

Automatic performance evaluation of a user carrying out a task has always been a challenge among researchers in both medical and nonmedical domains. Such evaluations are usually subjective and, in the real world, are performed by judges who are experts in the given field. For example, evaluation of the quality of a dance, a gymnastic performance, or a physiotherapy/rehabilitation exercise may be performed by expert dancers, sport athletes, and professional therapists, respectively. Such evaluations require the presence of human specialists who may not be easily accessible or affordable. In addition, the fact that the assessment is subjective indicates that a different expert might have a different opinion. The ability to conduct objective automatic evaluations that are repeatable is thus highly desirable. A real-world example is a video game called Just Dance, which is developed by the French company Ubisoft for Microsoft Xbox. Using the Kinect sensor, the players must mimic the onscreen dancer's choreography to a chosen song. The system then continuously evaluates in real time the quality of a user's dance movements in terms of being "Ok," "Good," "Super," or "Perfect" and reports a total numeric score at the end [22].

Studies in the literature concerned with automated evaluation of therapy motions are scarce [23-26], and not much attention has been paid to the development of metrics for performance evaluation [27]. As a common scheme, a reference model is first captured as the ground truth. Then, a user's performance can be compared with the reference using machine learning approaches. A comprehensive taxonomy of the metrics for evaluation of patient performance in physical therapy was proposed by Vakanski et al [27]. The metrics are classified into quantitative and qualitative categories. Further, quantitative metrics are divided into model less (based on raw measurements of motions) and model based (based on a mathematical model of the motions). Of the reviewed metrics, root mean square distance, Kullback Leibler divergence, log likelihood, and Fugl-Meyer assessment were used to classify a set of 5 human motions captured with a Kinect sensor.

Using KEHR, Su et al [19] applied DTW and fuzzy logic to detect real time subjective discrepancies between the model exercise and the performance of the patient. Before applying either algorithm, the user's execution of the prescribed exercise was recorded under the supervision of a professional. Then, 2 factors were included for the assessment: (1) trajectory disparity, the motional path created by each joint over time, and (2) speed variation, the time used to complete a designated exercise. Applying HMM and defining an accept/reject interval, a method to detect deviations from normal repetitions in therapeutic activities was presented by Palma et al [23]. The authors later compared the performance of their HMM-based technique with that of DTW [28]. A similar approach using HMMs to assess the correctness of telerehabilitation exercises was employed by Deters et al [24], whereas a cloud-based physical therapy monitoring and guidance system that applies DTW to produce subjective assessments in terms of being too slow/fast or overdone/incomplete was proposed by Wei et al [25]. Richter et al [26] presented an error classification algorithm for therapy exercises based on incremental DTW to classify the incorrect motions in a hip abduction exercise into 4 discrete categories: bent knee, foot outside, upper body, and wrong plane. A variance of DTW called multi-template, multi-match DTW was used by Yurtman and Barshan [29] to detect and evaluate physical therapy exercises using wearable motion sensors, providing a quantitative measure of similarity between an exercise execution and previously recorded templates. A continuous time warping algorithm based on automatic motion assessment learning was introduced by Tal and Shimshoni [30]. The resulting models produced numerical scores comparable with those of the Fugl-Meyer assessment. Recently, a DTW-based algorithm was developed for assessing Kinect-enabled home-based rehabilitation exercises to support auto coaching in a virtual gaming environment [31]. Using a simple but innovative method, the DTW distances are converted to meaningful performance scores in terms of percentage. By conducting a user study, the scores are then validated with the expert ratings showing a strong positive linear relationship. In another recent work, a deep learning-based framework for the assessment of rehabilitation exercises was proposed [32]. The framework consists of algorithms for dimensionality reduction, performance metrics (based on the Gaussian mixture model), scoring functions, and deep learning models. The authors demonstrated the capacity of the trained models by evaluating a data set of 10 rehabilitation exercises.

Similar techniques have also been employed in other domains, such as dance motion evaluation. Jang et al [33] employed DTW and Laban movement analysis to evaluate the correctness of dance movements in terms of being best, good, bad, and worst. In another work [34], the authors employed HMM for dance gesture recognition and evaluation, comparing the results with 
those of domain experts in terms of being good, medium, or bad.

A common factor among all these efforts was that they focused on evaluating the incorrectness of the performance on the basis of subjective terms. In most cases, the method developed was used to sort multiple erratic performances with respect to a reference template. This approach motivated us to explore the use of DTW and HMM to generate a similarity score between a participant's performance and a reference.

\section{Methods}

\section{Materials}

\section{MIRA}

MIRA is a software platform that turns physiotherapy exercises into clinical exergames [35]. It aims to increase engagement levels and improve the uptake of exercises by converting the rehabilitation sessions into entertaining activities, making therapy more convenient and easier to follow, and offering greater accessibility. In turn, this has the potential for shorter recovery times as well as supporting physiotherapists, reducing workload, and waiting times at clinics. MIRA has been used in several clinical studies [36-39].
The MIRA system includes a Kinect V2 sensor (Microsoft Corp) connected to a computer running the MIRA program (Figure 1). Currently, 32 exercises and 25 games are supported by MIRA. Each rehabilitation session requires selecting an exercise and a suitable game (Figure 2). Exercises include shoulder abduction, elbow flexion, and side strides, and examples of games include Firefly, Fishing, and Football. Once an exercise has been selected, adequate game options are presented. The selected combination of exercise and game is then added to the session and can subsequently be executed. Each execution starts with a process of calibrating the patient's position in front of the Kinect. A short video tutorial explaining the exercise is followed by another video tutorial describing the game mechanics. As the game starts, the user must play it by moving the intended body part (ie, left arm, right leg, or neck) in the manner shown in the video.

At the end of each session, the MIRA system reports various scores. Depending on the game, it reflects on the extent to which the player follows the game's objectives. For example, the number of fish caught and taken to the boat or the number of times the spaceship is safely passed through the fire rings. Although the scores can be an indication of how well the user played the game, they do not have much value in a clinical context. The aim of this work is to introduce an objective evaluation method that is more meaningful and suitable for clinical evaluation.

Figure 1. Medical Interactive Recovery Assistant system including a Kinect motion sensor and software to match an exercise with a game. A child (left) and an elderly gentleman (right) playing the Atlantis game. The child is practicing an arm exercise, the gentleman a hip exercise.
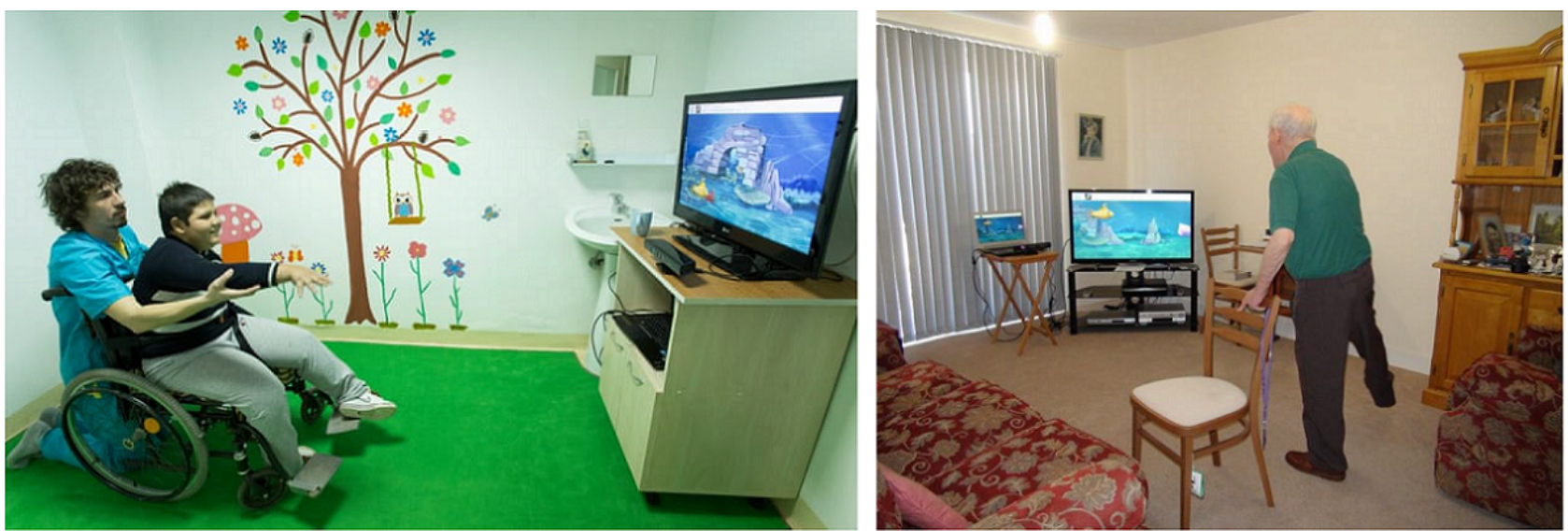
Figure 2. A snapshot of the Medical Interactive Recovery Assistant program. Exercises are listed on the left and games are shown on the right. Multiple game options allow practicing the same exercise with different games, thus encouraging patients to cope with the prescribed exercise by discovering the various game scenarios.

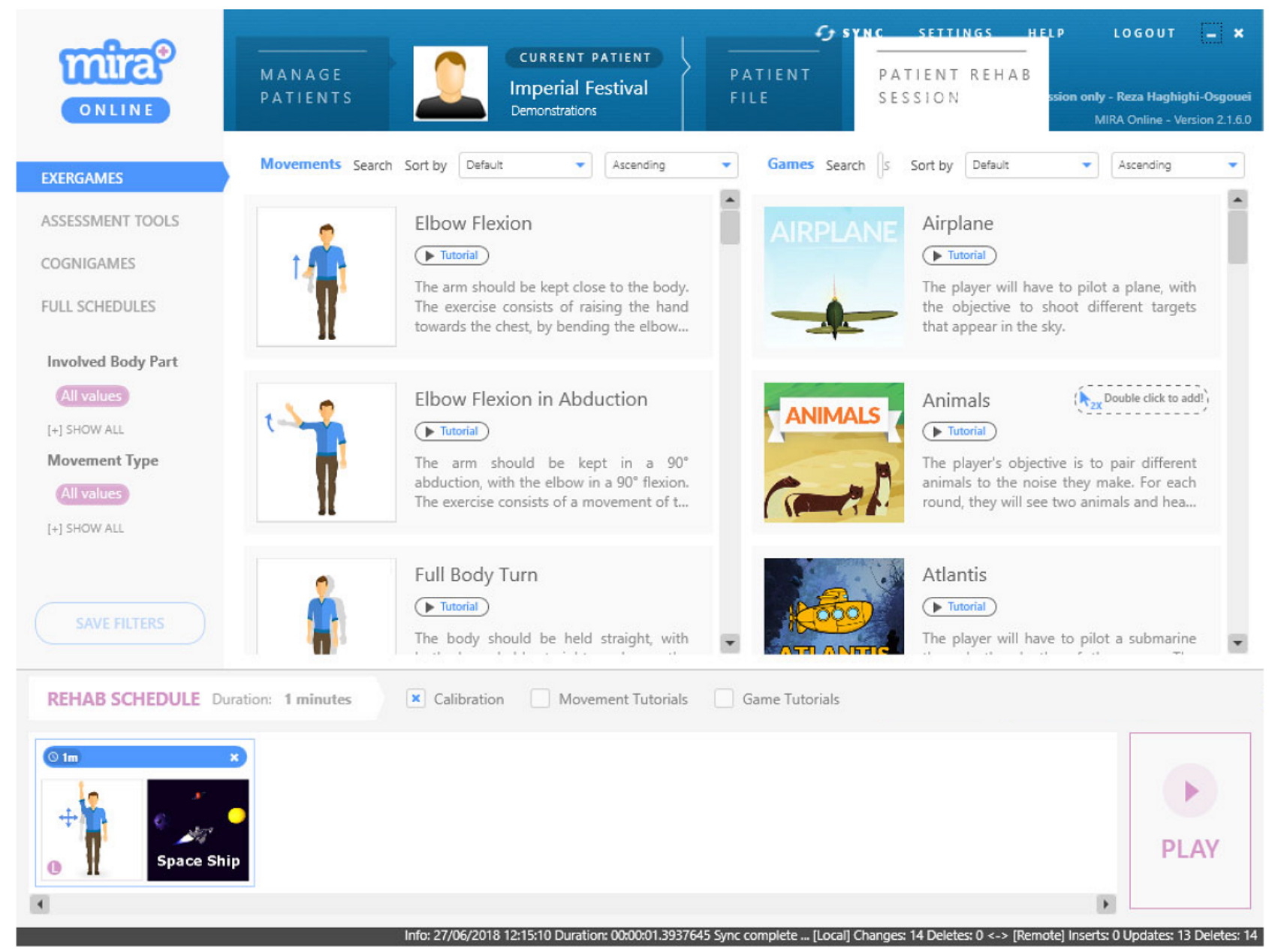

\section{Data Collection}

We developed a program in the Unity 3D game engine (Unity Technologies) [40] to capture and store raw 3D position coordinates of the selected joints using the Kinect. This was needed as the MIRA program does not allow accessing joint data when playing an exergame because of regulations imposed on class I medical devices. There were no technical problems executing both programs in parallel as the Kinect SDK permits simultaneous access to the sensor from multiple sources. Using Kinect V2, the 3D position coordinates of 25 different human skeleton joints can be tracked with an update rate of $30 \mathrm{fps}$. However, it is not necessary to track all the joints but only those that are involved in the chosen exercise. For this study, 4 different types of exercises were selected in consultation with a physiotherapist (DS): shoulder abduction (both left and right arms), hip abduction (both left and right legs), lunge (both left and right legs), and sit-to-stand exercises. This resulted in a total of 7 exercises to be performed by participants. A brief description of each exercise is given in Textbox 1.

As 3D position coordinates are dependent on the user size and location in front of the Kinect camera, we decided to extract invariant features (joint angles) to describe each exercise optimally (Figure 3). These scalar features were discussed with the physiotherapist and confirmed to be sufficient for capturing the essence of each exercise. For example, in shoulder abduction, the shoulder angle $\left(\theta_{1}\right)$ reflects the range of motion and the arm angle $\left(\theta_{2}\right)$ indicates whether the arm is being stretched or not. Except the lunge exercise, which required 3 joint angles, all other exercises could be described with only 2 angles. A sample plot of the extracted features is shown in Figure 4.

A motion trajectory $\mathrm{T}(\mathrm{l})$ is formed by the sequence of feature values within the time frame $0 \leq t \leq 1$, where 1 is the execution time. $T(1)$ is a matrix of size $1 \times 3$ for the lunge exercise and $1 \times 2$ for rest.

$$
T(l)=\left[\begin{array}{c}
\theta_{1}(0), \theta_{2}(0) \\
\theta_{1}(1), \theta_{2}(1) \\
\vdots \\
\theta_{1}(l), \theta_{2}(l)
\end{array}\right]
$$


Textbox 1. Correct execution of each exercise.

- Shoulder abduction: the arm should be kept close to the body. The exercise consists of raising the arm away from the side, keeping it in a straight line with the body.

- Hip abduction: the leg should be held straight and on the ground. The exercise involves raising the leg away from the side, keeping it in a straight line with the body.

- Lunge: stand straight facing forward with the spine and the pelvis in a neutral position. Take a step forward with a leg that is long enough so that when the knee bends, it does not go beyond the toes. Bend the back knee until it almost touches the floor, keeping both the torso and the spine in a neutral position. Return to the starting position.

- Sit-to-stand: sit on a chair. Without using the hands for support, stand up and sit back down. Make sure each movement is slow and controlled.

Figure 3. Extracted features (joint angles) from the joint 3D positions for each type of exercise. Both 2D side view and 3D perspective view are provided for clarity. 2D: 2-dimensional; 3D: 3-dimensional.

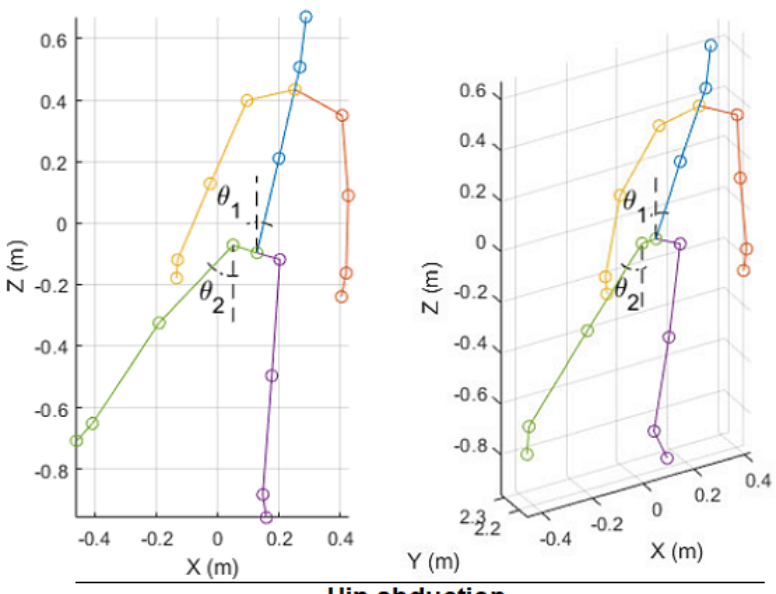

Hip abduction

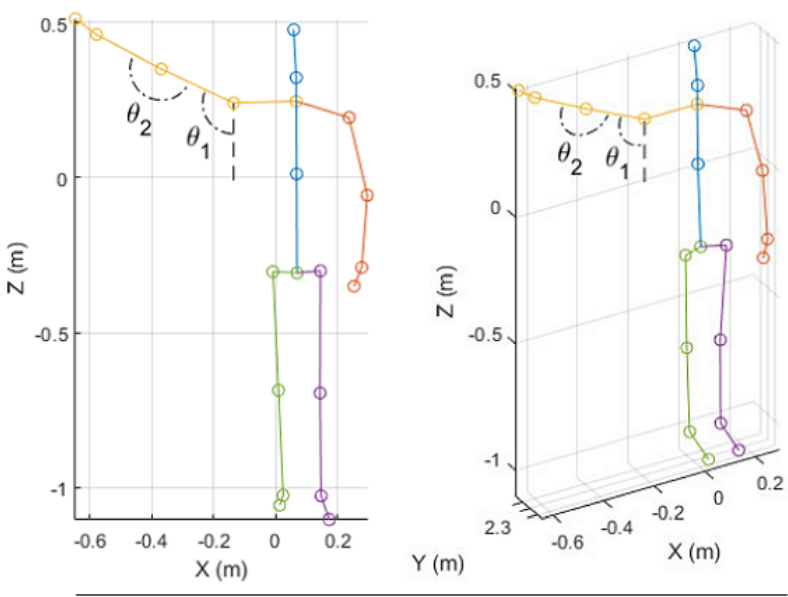

Shoulder abduction
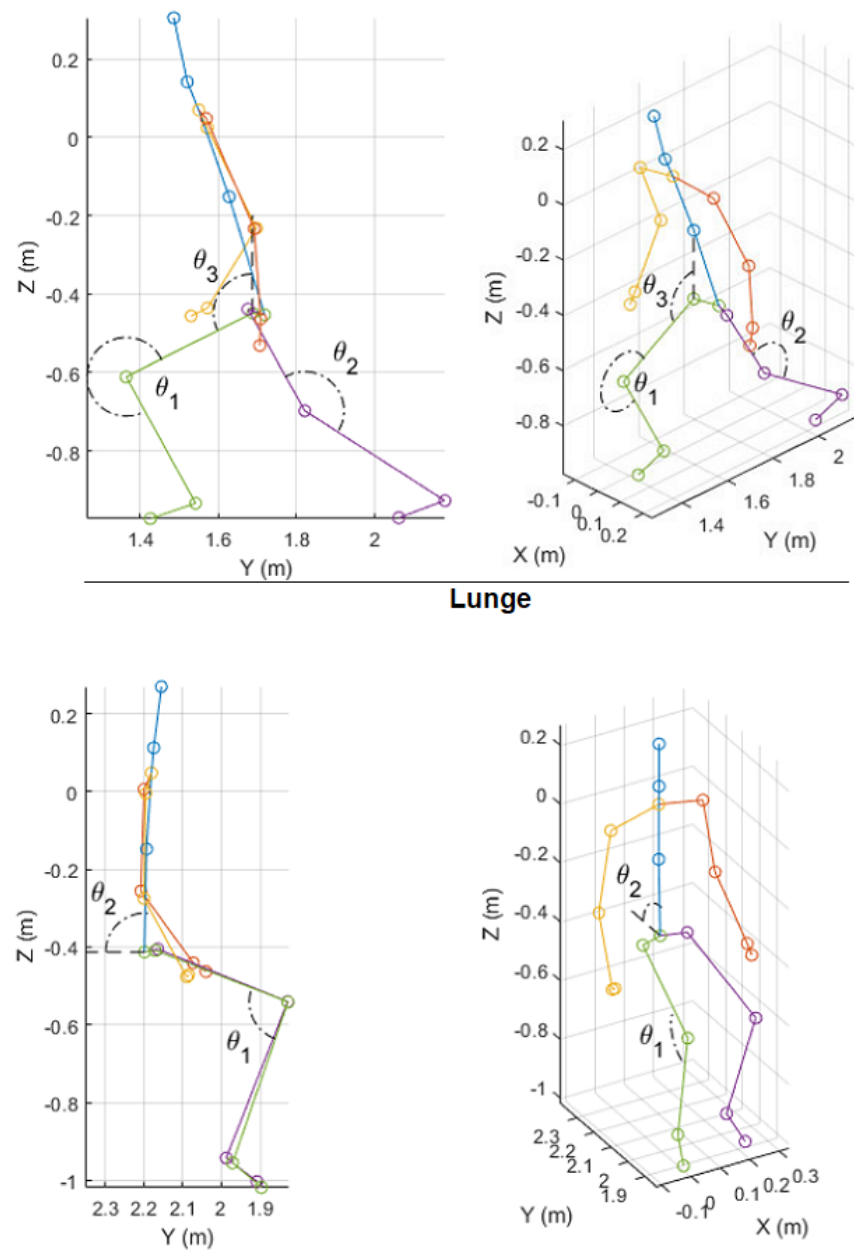

Sit-to-stand 
Figure 4. Sample extracted features obtained from 3D position for all seven exercises: hip abduction left (hpl) and right (hpr), lunge left (lul) and right (lur), shoulder abduction left (shl) and right (shr), and sit-to-stand (sit).
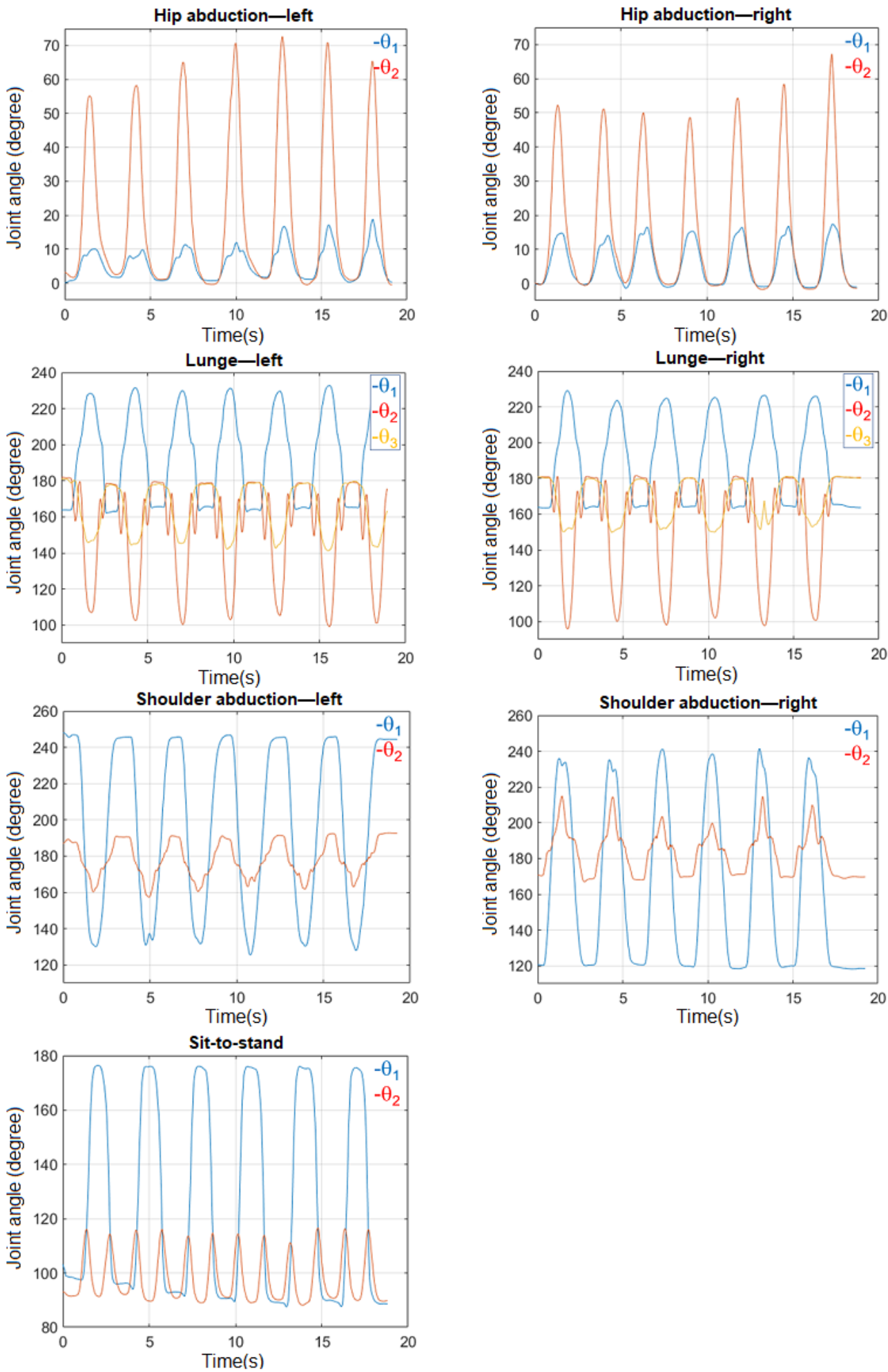

\section{DTW}

DTW [41] is a technique that aligns 2 time series and determines the minimum Euclidean distance between them. It is a frequently used approach in speech recognition to classify sound waves of the same word spoken in different accents and durations. DTW is sensitive to both the signal pattern and the amplitude. If 2 signals have the same patterns, for example, the same

number of peaks but different amplitude, then the alignment cannot be perfect, thus yielding a large distance between them. If they have the same amplitude but different patterns, the alignment will also result in a large distance. Therefore, the output distance is a measure of the similarity between 2 time series. The higher the distance, the greater the deviation.

Although DTW was initially applied to speech recognition, it has also been widely used in gesture recognition $[10,12,42]$. In 
these studies, the motion trajectories, each representing a gesture, are classified into the most similar gesture group (ie, the one with the smallest distance) by converting the distance between 2 trajectories into a similarity measure.

We define $\mathrm{D}_{\mathrm{RP}}=\mathrm{DTW}\left(\mathrm{T}_{\mathrm{R}}, \mathrm{T}_{\mathrm{P}}\right)$ as a distance measure between the reference $\left(T_{R}\right)$ and the participant $\left(T_{P}\right)$ trajectories. The MATLAB function $d t w$ was used for the implementation. Although the lower limit $\left(D_{1}\right)$ of this distance is 0 (being perfectly similar), the upper limit is unknown and can take any large value. By estimating an upper limit, it is possible to convert the distance measure into a similarity score. By associating the upper bound with the worst possible performance, an upper limit can be approximated. For all the exercises, not moving would be the worst performance. We refer to this worst trajectory as $\mathrm{T}_{\mathrm{W}}$. Calculating $\mathrm{D}_{\mathrm{RW}}=\mathrm{DTW}\left(\mathrm{T}_{\mathrm{R}}, \mathrm{T}_{\mathrm{W}}\right)$ allows us to establish the upper bound. Knowing both the lower and the upper bounds, a given distance $D_{1}=0 \leq D \leq D_{u}$ can be transformed into a similarity measure or percentage score $0 \leq S_{D} \leq 100$ :

$$
\mathrm{S}_{\mathrm{D}}=100 \times\left(\mathrm{D}-\mathrm{D}_{1}\right) /\left(\mathrm{D}_{\mathrm{u}}-\mathrm{D}_{1}\right)=100 \times\left(\mathrm{D} / \mathrm{D}_{\mathrm{u}}\right)
$$

\section{HMM}

An HMM [43] is a stochastic model that considers an observed signal as the result of the transition of a system between several states, each of which has the probability that a particular symbol might be observed. HMMs are useful for the recognition of temporal patterns such as speech, handwriting, and gestures. An HMM with discrete observations is mainly specified by the state transition matrix A and the observation matrix B, assuming that the system goes through $\mathrm{N}$ different possible states $\mathrm{S}_{1}, \mathrm{~S}_{2}$, $\ldots, S_{\mathrm{N}}$ and in each state, one of $\mathrm{M}$ different symbols $\mathrm{v}_{1}, \mathrm{v}_{2}, \ldots$, $\mathrm{v}_{\mathrm{M}}$ can be observed (Figure 5).

For performance evaluation, a single $\mathrm{HMM}, \lambda_{\mathrm{R}}$, is trained based on the reference motion trajectory $T_{R}$. We then calculate the $\log$ likelihood of $T_{P}$ given the trained model by $\mathrm{L}_{\mathrm{RP}}=\log \left(\mathrm{P}\left(\mathrm{T}_{\mathrm{p}} \mid \lambda_{\mathrm{R}}\right)\right) / 1_{\mathrm{p}}$. Similar to DTW, the lower and upper limits of the log likelihood need to be calculated. The upper limit $\left(\mathrm{L}_{\mathrm{u}}\right)$ is known and is equal to 0 , as the highest probability is 1 . However, the lower limit is unknown and can be any small value less than zero. Same as before, we assumed that this lower limit reflects the worst possible performance captured by $T_{W}$. Letting the lower limit be $\mathrm{L}_{\mathrm{RW}}=\log \left(\mathrm{P}\left(\mathrm{T}_{\mathrm{w}} \mid \lambda_{\mathrm{R}}\right)\right) / 1_{\mathrm{w}}$, the similarity score $0 \leq \mathrm{S}_{\mathrm{H}} \leq 100$ corresponding to $\log$ likelihood $\mathrm{L}_{\mathrm{l}} \leq \mathrm{L} \leq \mathrm{L}_{\mathrm{u}}=0$ is obtained by:

$$
\mathrm{S}_{\mathrm{H}}=100 \times\left(\mathrm{L}_{-} \mathrm{L}_{\mathrm{l}}\right) /\left(\mathrm{L}_{\mathrm{u}}-\mathrm{L}_{\mathrm{l}}\right)=100 \times\left(\mathrm{L}_{\mathrm{l}}-\mathrm{L} / \mathrm{L}_{\mathrm{l}}\right.
$$

Figure 5. Hidden Markov model with three states (q1 to q3) and five observation symbols (v1 to v5). The relationship among states is described by transition matrix $\mathrm{A}=[\mathrm{aij}] 3 \times 3$, and between states and discreet symbols by observation matrix $\mathrm{B}=[\mathrm{bij}] 3 \times 5$. It is assumed the system evolves through certain states whose relationship is to be studied.

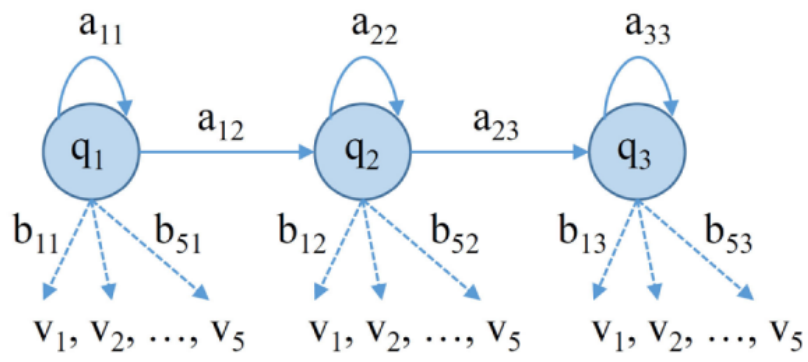

\section{Experimentation}

\section{Setup}

Standard hardware (computer, television, Kinect sensor) was used in combination with a Unity program to display the participant's live performance on the screen and store the 3D position data along with a time stamp. As mentioned above, 4 types of exercises were chosen to be performed by the participants: shoulder abduction, hip abduction, lunge, and sit-to-stand exercise. Except the sit-to-stand exercises, all other exercises were performed for both the left and the right sides, resulting in a total of 7 exercises.

\section{Participants}

A total of 16 healthy participants, including 8 adult females (22 to 30 years), 6 adult males ( 22 to 40 years), and 2 school boys (12 and 17 years), were recruited for the study. Participants were asked to stand in front of the Kinect sensor and perform each of the 7 exercises for 20 seconds. They were told to repeat the chosen exercise at least five times with a short pause between each repetition. In addition to the 16 participants, the physiotherapist involved in the project (DS) was asked to perform the exercises as the reference performance. He repeated each of the 7 exercises at least five times during a period of 20 seconds each.

\section{Results}

Of the 5 repetitions, 3 were extracted (ignoring the first and the last) for each participant. For DTW scores, the distance between each repetition of a participant and each repetition of the physiotherapist was calculated, yielding a total of 9 values. The final DTW score $\left(\mathrm{S}_{\mathrm{D}}\right)$ was obtained by taking the average of these values. For HMM scores, the likelihood of each repetition of a participant given the physiotherapist's model was calculated, yielding a total of 3 values. The final HMM score $\left(\mathrm{S}_{\mathrm{H}}\right)$ was obtained by taking the average of these values. Figure 6 shows the similarity scores obtained by applying DTW $\left(\mathrm{S}_{\mathrm{D}}\right.$, solid blue line) and HMM ( $\mathrm{S}_{\mathrm{H}}$, solid red line) for each exercise. The difference between the 2 scores, $S_{D}-S_{H}$, is also indicated in the plots by a dashed black line. Participant 17 is the 
physiotherapist and hence the reference. As expected, he obtained the maximum score in all cases. The worst performance, that is, making no movement, is included as participant 18 , yielding very low scores in all exercises. Ideally, we would have expected to obtain a maximum score of $100 \%$ for the reference and a minimum score of $0 \%$ for the worst performance. However, these values were not obtained because of natural variability among the repetitions and cross-comparison between the repetitions.

Figure 6. Similarity scores obtained from applying dynamic time warping (SD, solid blue line) and hidden Markov model (SH, solid red line). Their difference, SH-SD, is shown by the black dashed line. Participant 17 is the physiotherapist and 18 is the worst performance, that is, making no movement. Error bars indicate standard error.
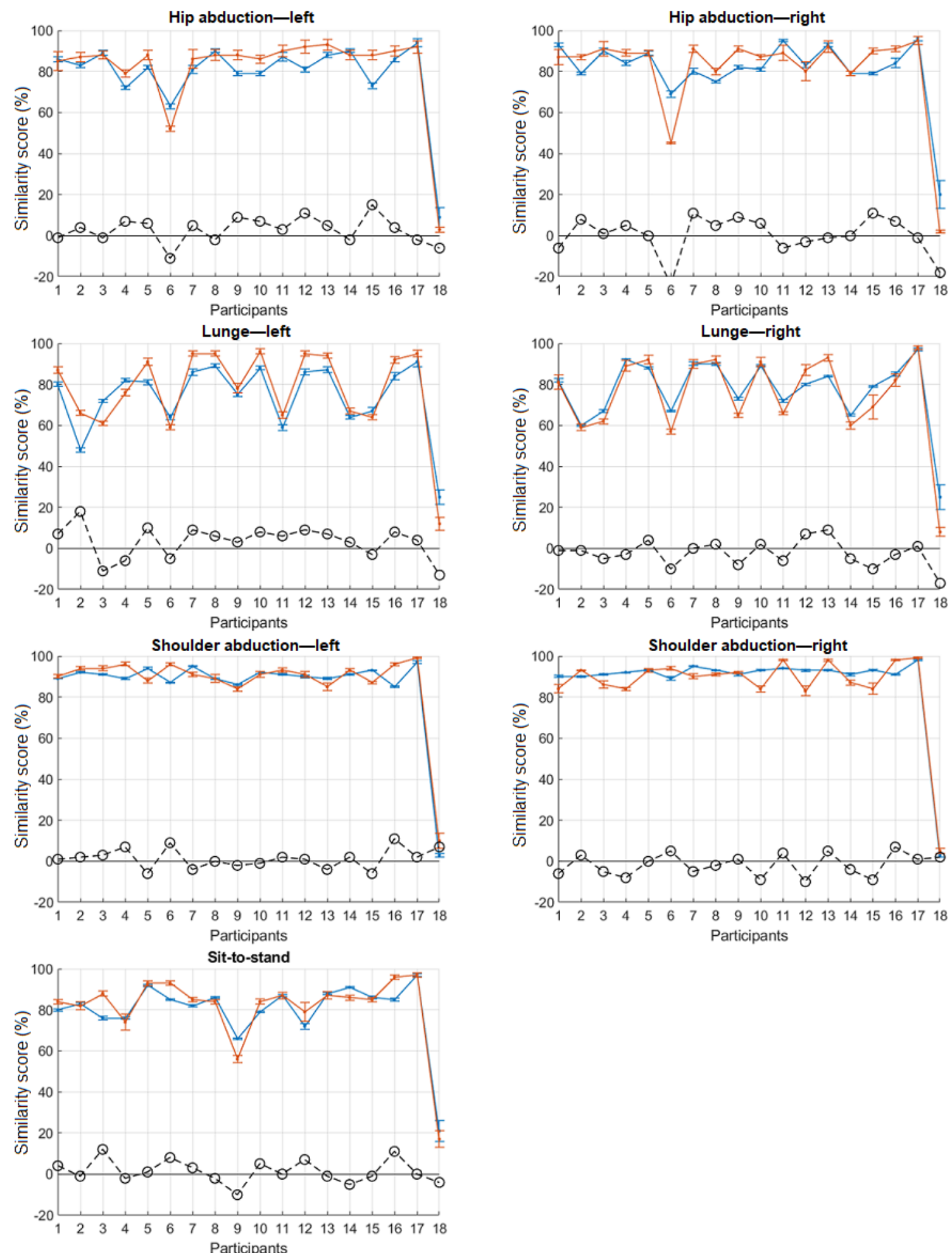


\section{Discussion}

Several observations can be made from these plots in Figure 6. Both scores showed similar trends. Whenever a participant did not perform well according to the DTW score $\left(\mathrm{S}_{\mathrm{D}}\right)$, his/her HMM score $\left(\mathrm{S}_{\mathrm{H}}\right)$ was low as well. Examples of this behavior are participant 6 in hip abduction (left and right) and participant 9 in sit-to-stand.

Regarding the difference, $\mathrm{S}_{\mathrm{H}}-\mathrm{S}_{\mathrm{D}}$, it is difficult to observe any obvious pattern. On some occasions, the difference was positive and on some others it was negative. There were also cases where the difference was negligible. The difference could be as large as $+18 \%$ (participant 2, lunge-left) or as small as $-24 \%$ (participant 6, hip abduction-right). For the reference participant 17 , the difference was generally very low $(-1 \%$, $-1 \%, 4 \%, 1 \%, 2 \%, 1 \%$, and $0 \%)$. However, this was not the case for the worst performance, participant 18. In most exercises, the difference was negative and was not negligible, indicating that the $S_{D}$ was usually larger than the $S_{H}$ for the worst performance. In addition, in most plots, $\mathrm{S}_{\mathrm{H}}$ was closer to $0 \%$ than $S_{D}$ for the worst performance.

Among the exercises, shoulder abduction was less challenging and easy to perform, which is reflected by participants performing well and achieving high similarity scores. In contrast, lunge was the most difficult and demanding exercise to perform, which is also reflected in the obtained similarity values.

Time domain plots of the best and worst performances can be used to visually examine the correlation between the trajectories and the calculated scores. For each of the 7 exercises, the trajectories of the best and the worst performances were plotted against the reference (Figure 7). Each plot illustrates 3 repetitions of the chosen participant (solid red lines) and 3 repetitions of the physiotherapist (dashed blue lines). It should be noted that for hip/shoulder abduction and sit-to-stand, each repetition includes 2 features, and for lunge, each repetition includes 3 features (Figure 4). Evidently, whenever the participant's trajectories match those of the reference (in terms of both amplitude and duration), the obtained score is high. Conversely, when the patterns do not match, the score is low. For example, in hip abduction-left, participant 6 failed to achieve full range of motion (compare the peak amplitude, $40^{\circ}$ versus $80^{\circ}$ ) and performed faster (compare the duration, 100 [1 second] versus 200 [2 seconds]). It is worth mentioning that both the DTW and the HMM algorithms are sensitive to amplitude than duration. They are both time-series algorithms that take into account the sequence of data (amplitudes) regardless of their frequency (duration). DTW does not allow time scaling of members within the sequence, and the HMM algorithm discards the time dependency of members by grouping them into clusters during the quantization preprocess. If two participants followed the required range of motion, but one performed slower (or faster) than the other, their score would be the same (see lunge-left, both participants 11 and 9).

Except the lunge exercise for which 3 features (joint angles) were extracted, 2 features were obtained for all the other exercises. However, not all the extracted features had the same weight and importance. For instance, in hip abduction, referring to Figures 4 and $7, \theta_{2}$ (hip angle) is the more relevant feature and $\theta_{1}$ (torso angle) had lesser importance. This is contrary to the results for shoulder abduction and sit-to-stand. For lunge, $\theta_{2}$ and $\theta_{1}$ were both equally important, but $\theta_{3}$ was lower. The main feature has a higher influence on the obtained similarity score. This is shown in Figure 8 for hip abduction-left as an example. Generally, the same trend was maintained for each measure (compare the same color solid and dashed lines). However, the effect of removing a minor feature $\theta\left({ }_{1}\right)$ was less influential on $S_{H}$ than on $S_{D}$, as DTW is more sensitive to detail than HMM. 
Figure 7. Time-domain plots of the best and worst trajectories (excluding participants 17 and 18). X-axis indicates the time in seconds and Y-axis indicates the joint angle in degree. The three repetitions of the reference trajectories are given in dashed blue lines and the chosen participant's trajectories in solid red lines. All plots include two features, except for lunge where three features are presented.

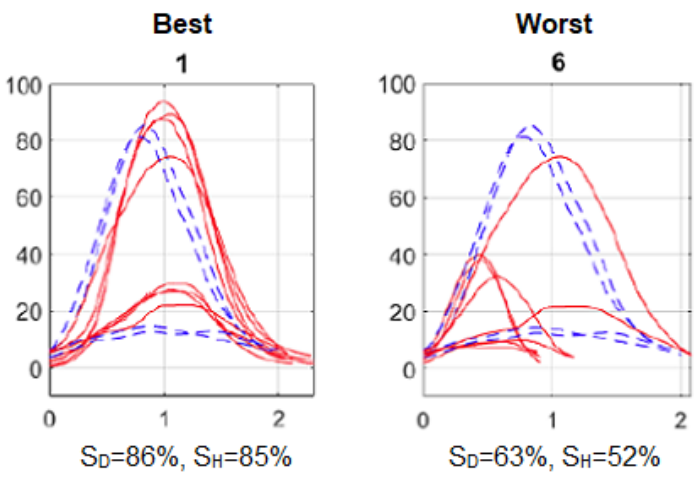

Hip abduction-left
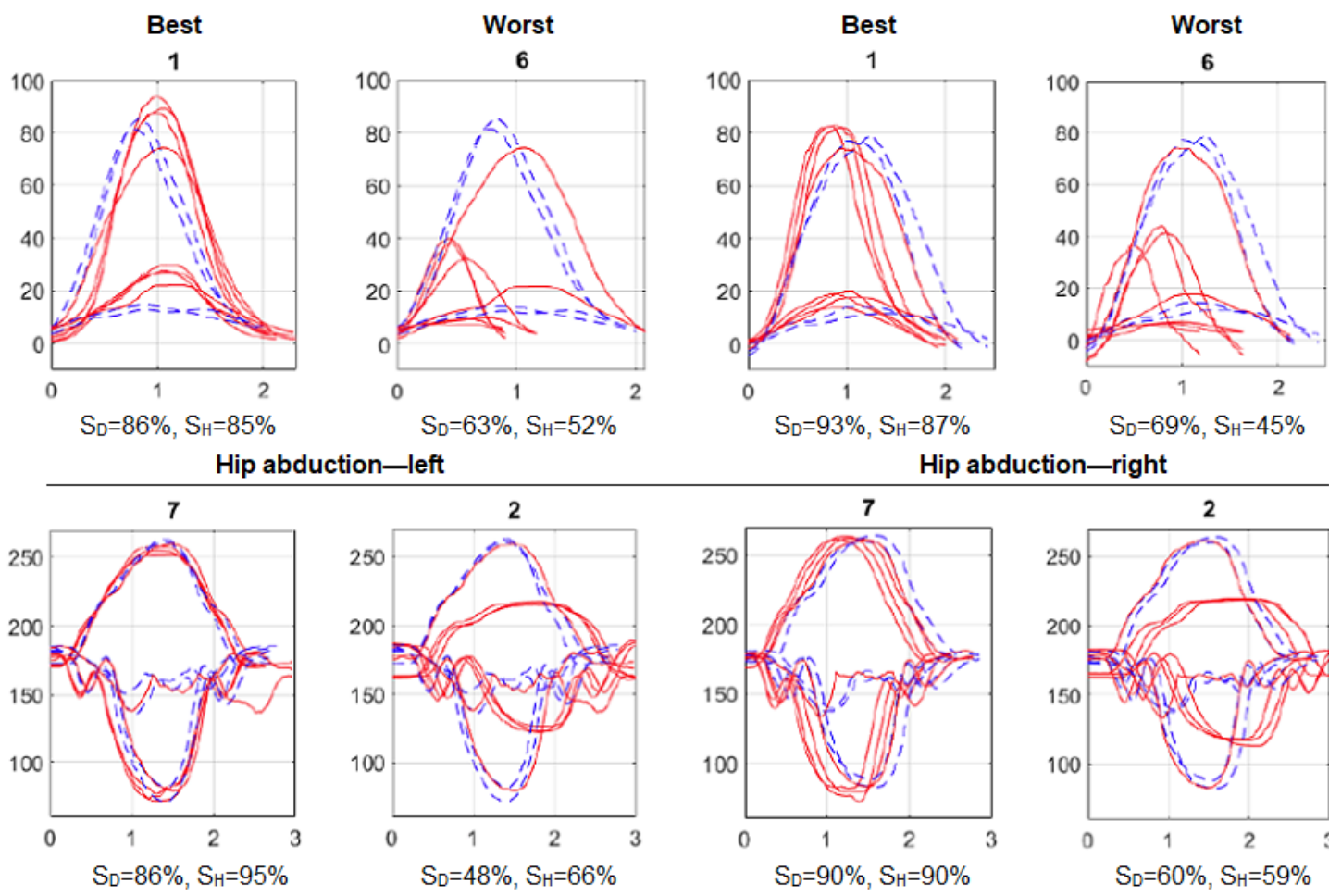

Hip abduction-right
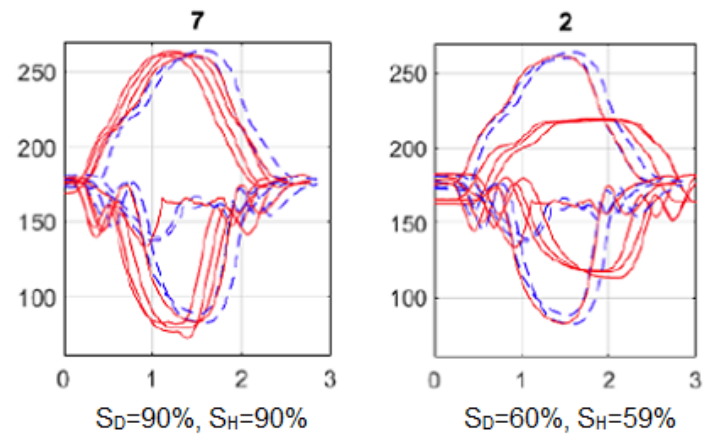

Lunge-left

Lunge-right
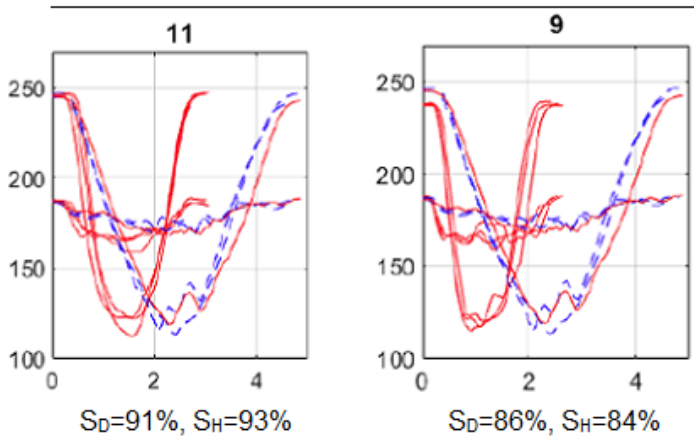

Shoulder abduction-left
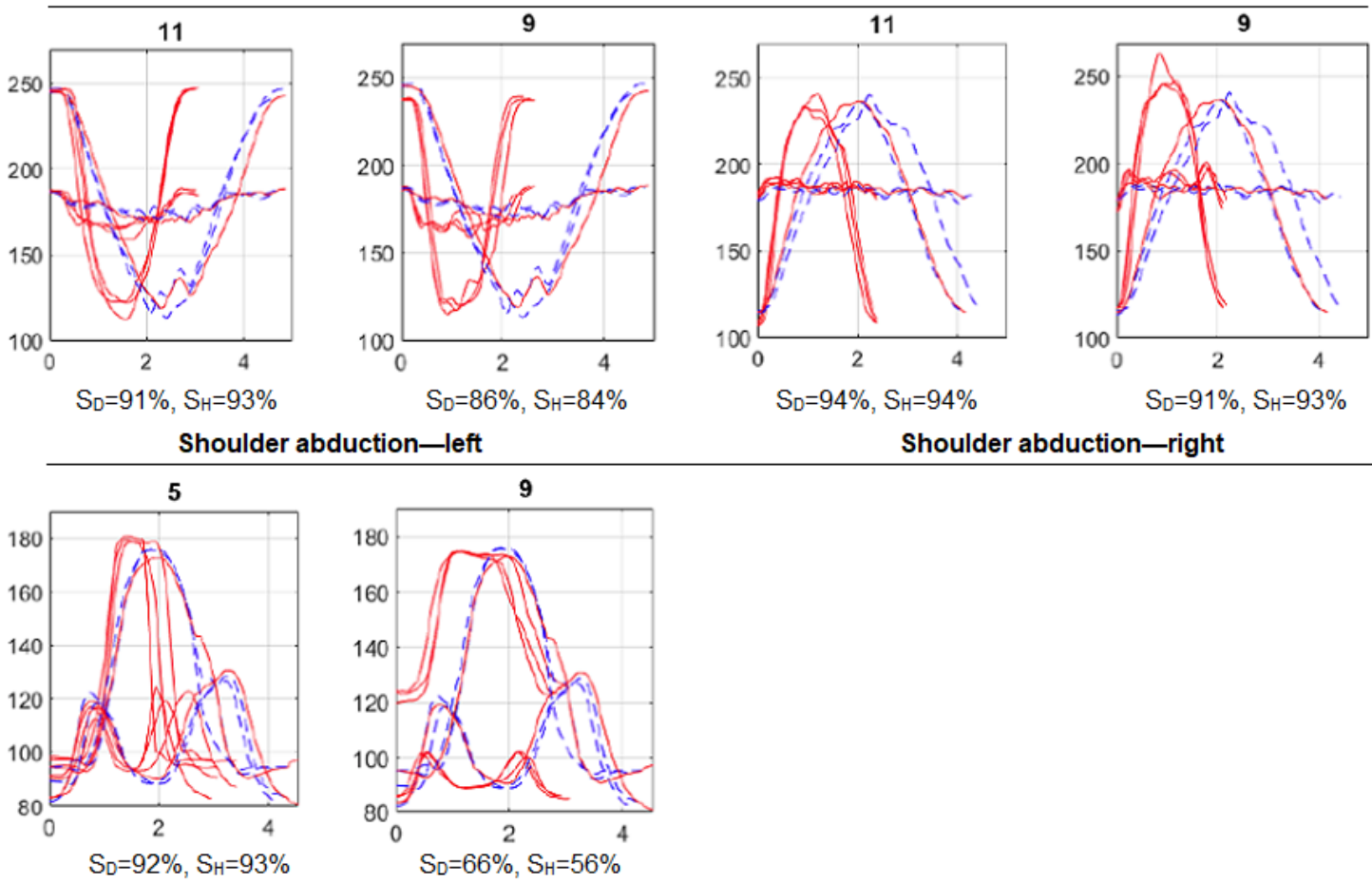

Shoulder abduction-right

Sit-to-stand 
Figure 8. The effect on the scores of removing minor features.

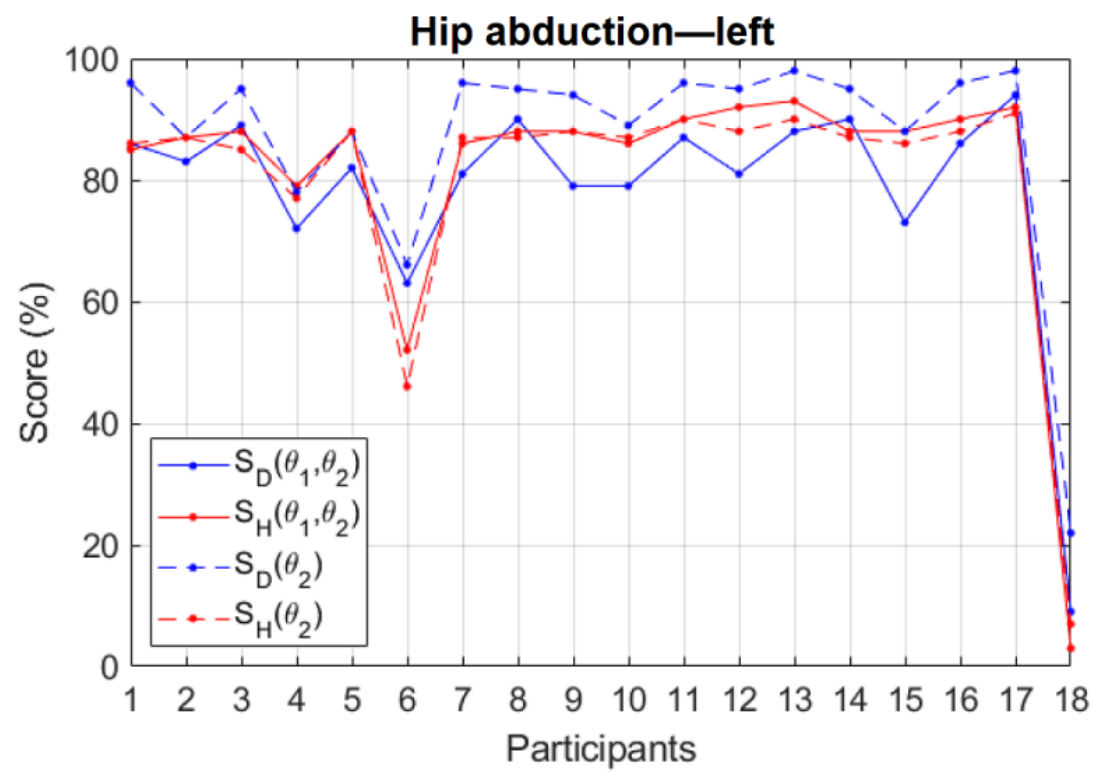

Although single-feature $S_{D}$ is clearly larger than full-feature $S_{D}$, single-feature $S_{H}$ is almost the same as full-feature $S_{H}$. Adding more details, that is, presenting additional minor features, increases the distance values (and hence decreases the similarity scores) obtained by applying DTW.

Although DTW is more sensitive to detail, HMM is more sensitive to the way the feature space is quantized. Quantization is a preprocess applied over the extracted features to segment them into several clusters for the purpose of training a discrete HMM. The boundaries and the number of clusters have an obvious effect on the HMM scores. This can be seen in Figure 9 for shoulder abduction-right as an example. Bivariate histogram plots are used to visualize the clusters. Three cases were tested, changing $\theta_{1}$ (main feature) boundaries and keeping $\theta_{2}$ (minor feature) intact. $\theta_{1}$ is divided into 3 clusters $\left(90^{\circ}, 180^{\circ}\right.$, and $\left.270^{\circ}\right)$ in case $\mathrm{A}, 4$ clusters $\left(90^{\circ}, 150^{\circ}, 210^{\circ}\right.$, and $\left.270^{\circ}\right)$ in case $\mathrm{B}$, and 5 clusters $\left(90^{\circ}, 135^{\circ}, 180^{\circ}, 225^{\circ}\right.$, and $\left.270^{\circ}\right)$ in case C. In all cases, $\theta_{2}$ is divided into 4 clusters $\left(90^{\circ}, 150^{\circ}, 210^{\circ}\right.$, and $270^{\circ}$ ). A closer look reveals that the $S_{H}$ scores in case $B$ are clearly lower than those in cases $\mathrm{A}$ and $\mathrm{C}$, which are more similar. The reason for this is that feature points are grouped into different clusters for the participant and the reference. This affects (reduces) the probability that a selected feature point of the participant is generated by the reference model. Most likely, the feature points of the participants and the reference in cases $\mathrm{A}$ and $\mathrm{C}$ are grouped into similar clusters; hence, their similarity scores are higher and more similar. In case B, however, they are grouped into different clusters, decreasing the probability values, thus lowering the similarity scores. One can take advantage of this behavior by adjusting the sensitivity of HMM similarity scores to smoothen or sharpen the differences. 
Figure 9. The effect of quantization on the calculated hidden Markov model scores.

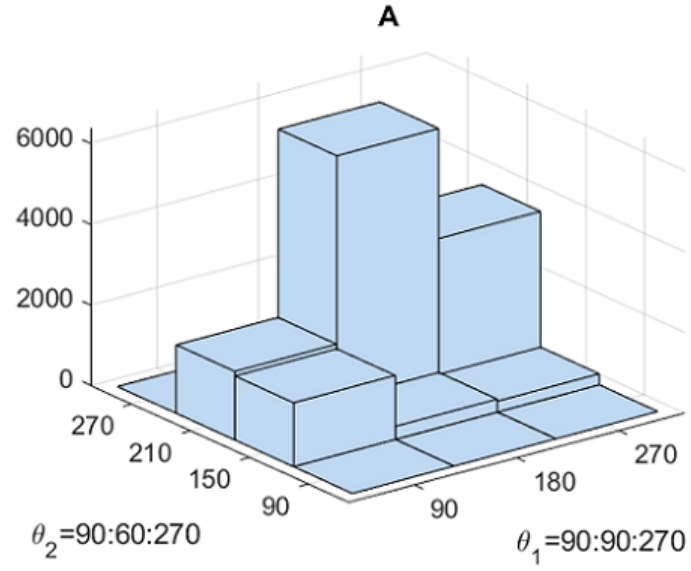

B

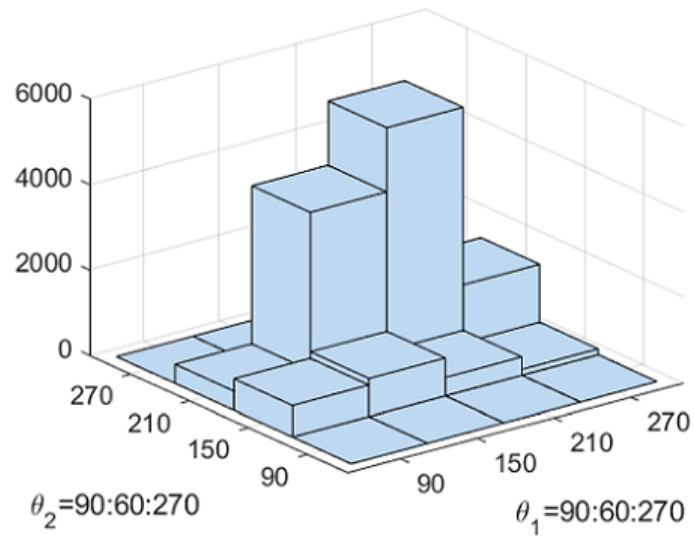

C

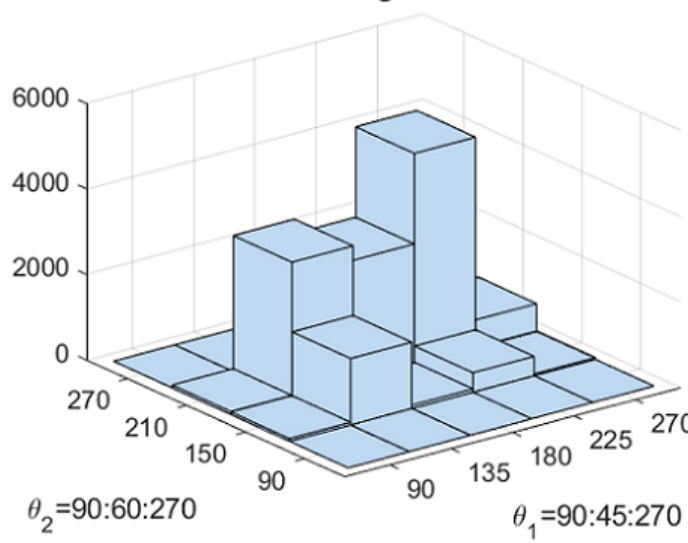

Worst performance is a key factor affecting the scores in both measures as it corresponds to the upper or lower boundary, as previously explained. This is evident from $\mathrm{S}_{\mathrm{D}}=100 \times\left(\mathrm{D}-\mathrm{D}_{1}\right) /\left(\mathrm{D}_{\mathrm{u}}-\mathrm{D}_{1}\right)=100 \times\left(\mathrm{D} / \mathrm{D}_{\mathrm{u}}\right)$ and $\mathrm{S}_{\mathrm{H}}=100 \times\left(\mathrm{L}_{-} \mathrm{L}_{1}\right) /$ $\left(\mathrm{L}_{\mathrm{u}}-\mathrm{L}_{\mathrm{l}}\right)=100 \times\left(\mathrm{L}_{\mathrm{l}}-\mathrm{L} / \mathrm{L}_{1}\right)$. Both unknown limits $\left(\mathrm{D}_{\mathrm{u}}\right.$ for DTW and $\mathrm{L}_{1}$ for $\mathrm{HMM}$ ) are used as denominators to normalize the distance $\mathrm{S}_{\mathrm{D}}=100 \times\left(\mathrm{D}-\mathrm{D}_{1}\right) /\left(\mathrm{D}_{\mathrm{u}}-\mathrm{D}_{1}\right)=100 \times\left(\mathrm{D} / \mathrm{D}_{\mathrm{u}}\right)$ and likelihood $\mathrm{S}_{\mathrm{H}}=$ $100 \times\left(\mathrm{L}_{-} \mathrm{L}_{1}\right) /\left(\mathrm{L}_{\mathrm{u}}-\mathrm{L}_{\mathrm{l}}\right)=100 \times\left(\mathrm{L}_{\mathrm{l}}-\mathrm{L} / \mathrm{L}_{\mathrm{l}}\right)$ values. The larger the denominator, the smaller the deviations (fluctuations) in the scores. This value can also be intentionally altered to adjust the sensitivity of the scores. With a larger denominator, the scores are smoother and the differences between participants become smaller. With a smaller denominator, the scores become sharper
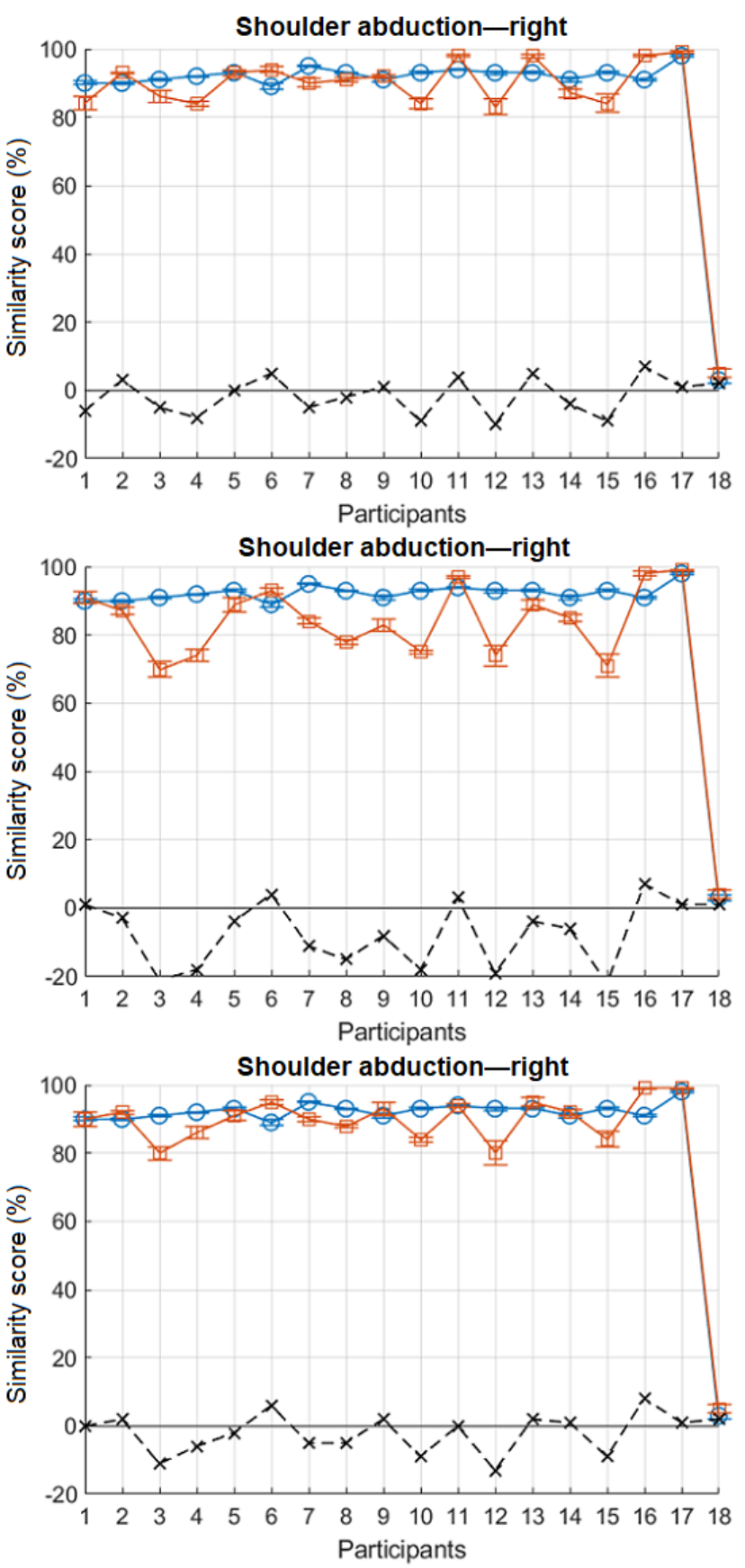

and the differences between participants are highlighted. As explained previously, we chose no movement as the worst performance for all exercises. Seemingly, a different worst performance can yield different scores if it generates different denominators. For example, one might say that closing the elbow in shoulder abduction could be worse than keeping it stretched (the current situation). An example of altering limits (multiplying and dividing $\mathrm{D}_{\mathrm{u}}$ and $\mathrm{L}_{\mathrm{l}}$ by 2 ) for sit-to-stand is shown in Figure 10. When the limits are magnified (multiplied by 2), the scores are increased and smoothed (Figure 10, left). When the limits are shrunk (divided by 2), the scores are decreased and sharpened (Figure 10, right). Smoothed scores can be used to encourage patients performing prescribed exercises in the early stages, whereas sharpened scores could 
be used in later stages to encourage further mastering of the skills involved in performing the exercises.

Several comparative tests were also conducted. Figure 11 shows the average scores across all 7 exercises. For each participant, the average score was obtained from $9 \times 7=72$ scores ( 9 values for each of the 7 exercises). As can be seen, the 2 plots show very similar trends. This was verified by applying the $t$ test over the 2 measures $(P=.49)$. Small error bars indicate high levels of consistency.
A comparison between the left and right performances, excluding sit-to-stand, is shown in Figure 12. No significant differences were observed between the left and the right scores. This was verified by applying a $t$ test over the scores for each measure ( $P$ values are given in Table 1$)$.

Furthermore, a comparison between female and male participants is shown in Multimedia Appendix 1. The scores were averaged across 8 female and 8 male participants (excluding reference). As it can be seen, there was no significant difference between the 2 genders. This was verified by applying a $t$ test over $\mathrm{S}_{\mathrm{D}}(P=.22)$ and $\mathrm{S}_{\mathrm{H}}(P=.86)$.

Figure 10. Effect of altering limits ( $\mathrm{Du}$ and $\mathrm{Ll}$, obtained from the worst performance) on the scores: multiplied by 2 (left) and divided by 2 (right). Error bars indicate standard error.
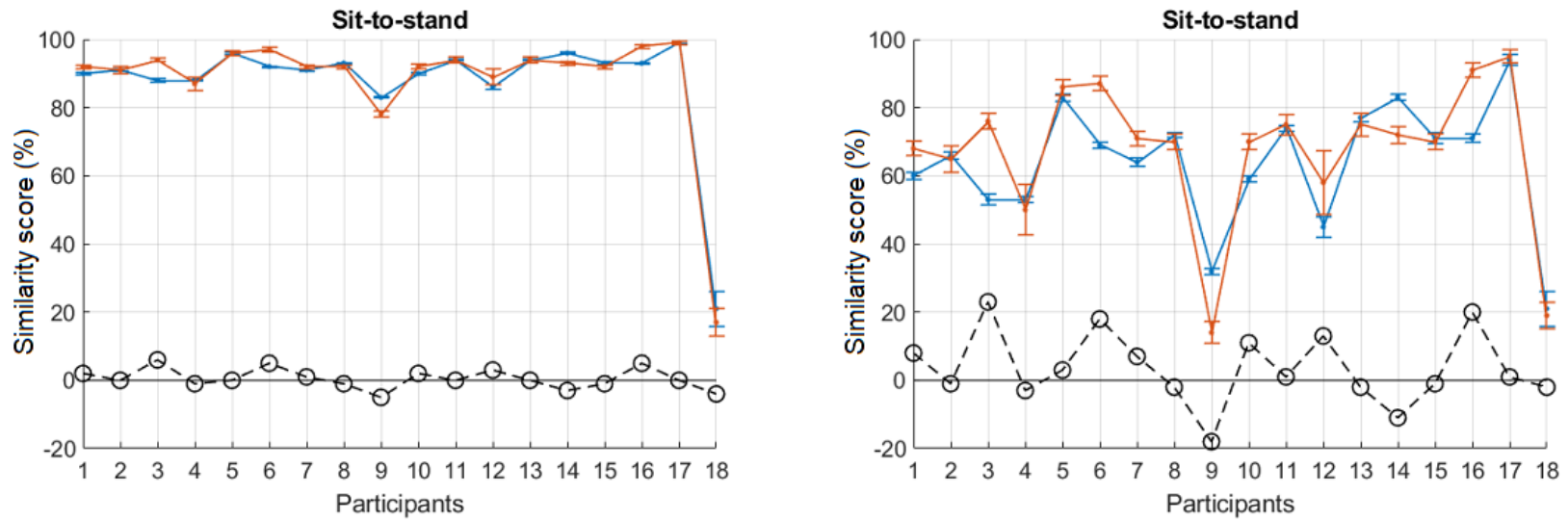

Figure 11. The combined and averaged scores (left: SD and right: $\mathrm{SH}$ ) for all seven exercises. Error bars indicate the standard error.
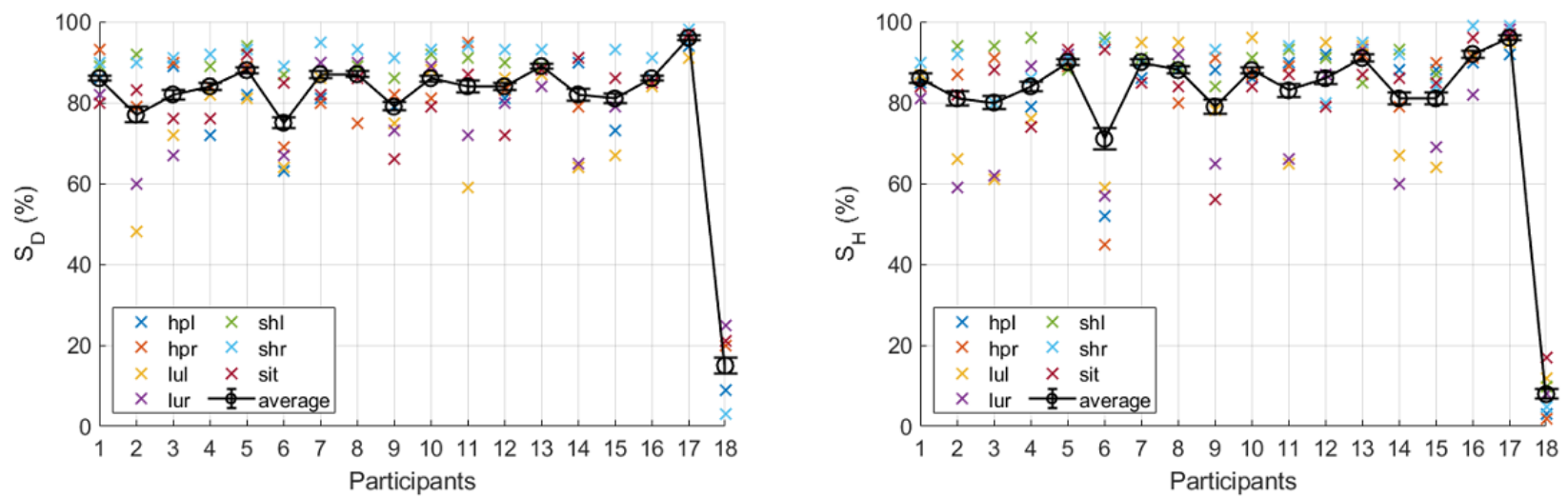
Figure 12. Comparison between left and right scores. Error bars indicate standard error.
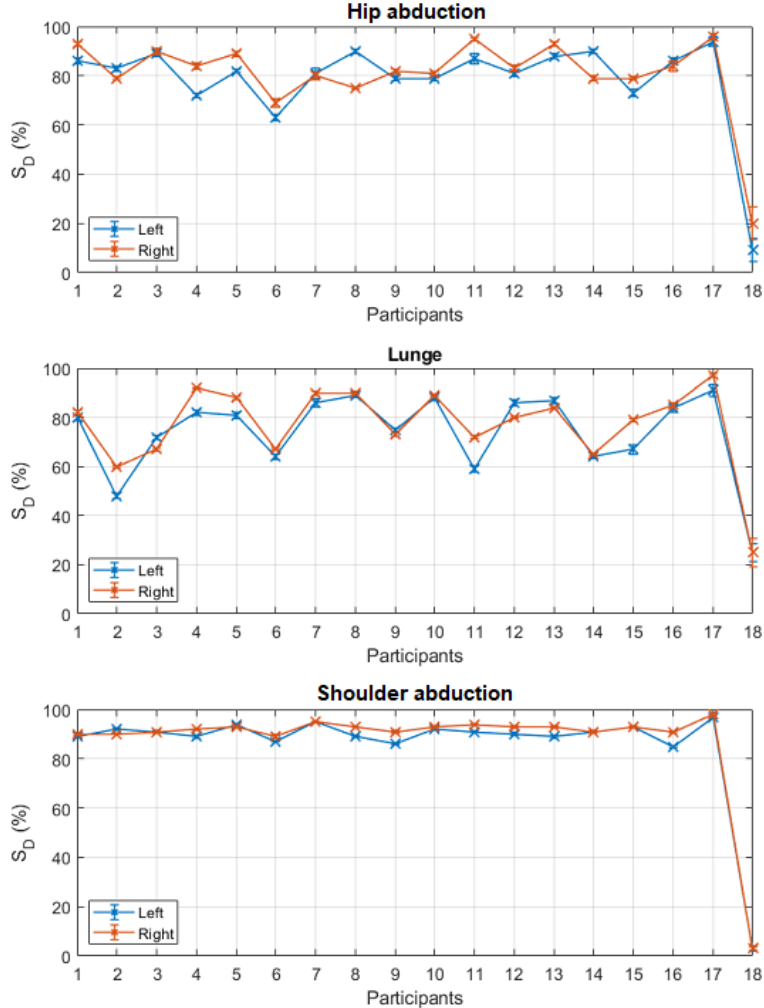
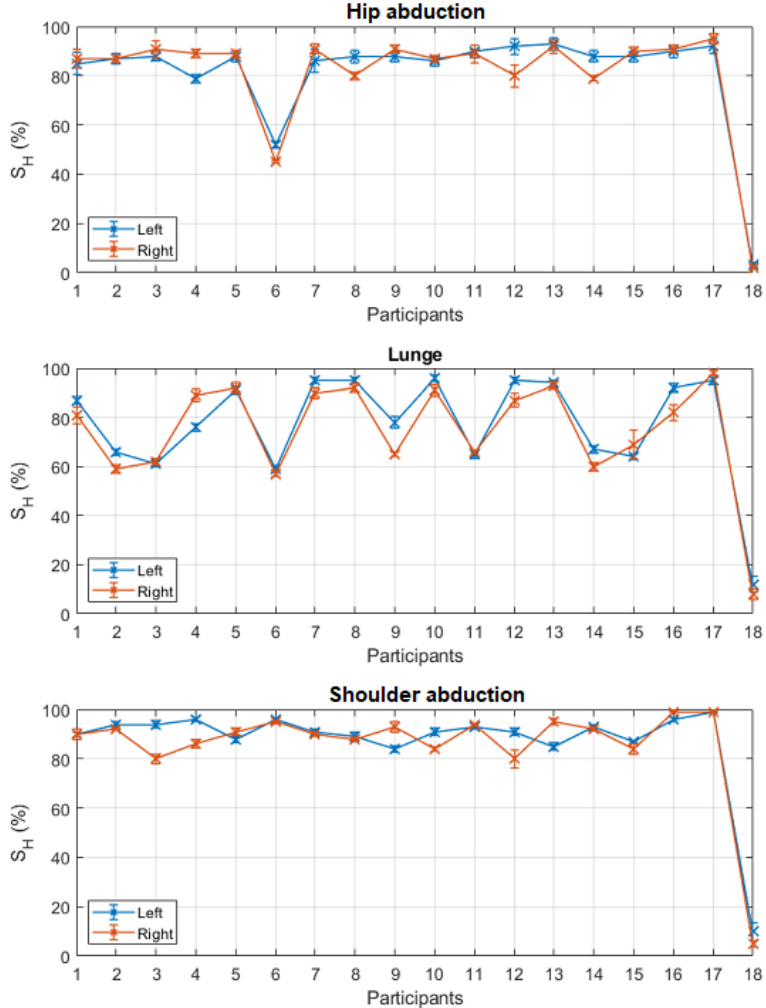

Table 1. $P$ values obtained by applying the $\mathrm{t}$ test over the left and right scores.

\begin{tabular}{llll}
\hline Metrices & Exercises & Lunge & Shoulder abduction \\
\hline $\mathrm{S}_{\mathrm{D}}{ }^{\mathrm{a}}$ & Hip abduction & .09 & .44 \\
$\mathrm{~S}_{\mathrm{H}}{ }^{\mathrm{b}}$ & .27 & .25 & .45 \\
\hline
\end{tabular}

${ }^{\mathrm{a}} \mathrm{S}_{\mathrm{D}}$ : dynamic time warping score.

${ }^{\mathrm{b}_{\mathrm{S}}} \mathrm{S}_{\mathrm{H}}$ hidden Markov model score.

\section{Conclusions and Future Remarks}

We implemented and compared 2 commonly used machine learning algorithms, DTW and HMM, to objectively evaluate the performance of patients using a rehabilitation exergaming platform. 3D movement data were obtained using the Kinect depth camera, and invariant features (joint angles) describing each exercise were extracted. The extracted features are independent of body fit, size, and position and distance of the user to the Kinect. They are also independent of the hardware being used and can be adapted for any motion-sensing device capable of tracking human skeleton joints, such as those mentioned in the Related Work section.

Setting a physiotherapist performance as the reference and making no movement as the worst performance, we applied both DTW and HMM algorithms to compare participants' performance and report a similarity score. The idea of worst performance was the key to converting the distance measures (obtained from DTW) and likelihood values (obtained from HMM) to similarity scores between $0 \%$ and $100 \%$. Overall, both algorithms showed similar trends but had different sensitivities. DTW was observed to be more sensitive to small changes, whereas HMM was more sensitive to the boundaries and clusters resulting from the quantization process. Both DTW and HMM are inherently more sensitive to range of motion than duration. In addition, both measures are sensitive to the worst performance. This suggests ways to use both algorithms to monitor patient progress at different stages: monitoring could start with HMM similarity scores in early stages for a more general comparison and switching to DTW similarity scores in later stages for finer comparison.

The application of these similarity scores is twofold. The scores can be used by the patients at home to encourage them to continue practicing the exergames to achieve higher similarity scores. In addition, the scores can be reported back to the physiotherapist to monitor patient progress and provide feedback. The exercise program can also be adjusted by the physiotherapist given the level of progress to better fit the patient's needs and progression. 
Our proposed method has the potential for significant impact in the context of rehabilitation exergames by enabling remote therapy home-based sessions where performance can still be adequately monitored. This can help better assess the quality of physical exercises performed by patients, fine-tune rehabilitation programs, and enhance the efficiency of home-based rehabilitation. In turn, cost reductions and freeing up of physiotherapy unit time may also be achieved.
Future work will include testing our proposed system on a public data set such as the University of Texas at Dallas-Multimodal Human Action Dataset [44]. In addition, we intend to recruit patients with reduced movement range or other constraints to participate in a study to validate our proposed performance metrics in a clinical setting. The study will also investigate the correlation between the objective scores used by our system and the subjective notes taken by physiotherapists when observing patients performing the exergames.

\section{Acknowledgments}

The project was funded through MedCity's Collaborate to Innovate program and received funding from the European Regional Development Fund and the Higher Education Funding Council for England. The authors acknowledge the support of MIRA Rehab Ltd as well as members of the Simulation and Modelling in Medicine and Surgery research group at the Imperial College London.

\section{Conflicts of Interest}

None declared.

\section{Multimedia Appendix 1}

Comparison between female and male participants, left: DTW scores (SD) and right: HMM scores (SH). Error bars indicate standard error.

[PNG File, 73 KB-Multimedia Appendix 1]

\section{References}

1. -. MIRA Rehab. URL: http://www.mirarehab.com [accessed 2020-07-15]

2. VirtualRehab. Evolv Rehabilitation Technologies. URL: https://evolvrehab.com/virtualrehab/ [accessed 2020-07-15]

3. Rehability: Rehabilitation Games Co-Designed With Patients. URL: http://rehability.me [accessed 2020-07-15]

4. Whitehead S, Johnston H, Nixon N, Welch J. Exergame Effectiveness: What the Numbers Can Tell Us. In: Proceedings of the 5th ACM SIGGRAPH Symposium on Video Games. 2010 Presented at: SandBox'10; July 26-30, 2010; Los Angeles, CA. [doi: $10.1145 / 1836135.1836144]$

5. Almeida J, Nunes F. The practical work of ensuring effective use of serious games in a rehabilitation clinic: a qualitative study. JMIR Rehabil Assist Technol 2020 Feb 21;7(1):e15428 [FREE Full text] [doi: 10.2196/15428] [Medline: 32130177$]$

6. Bossen D, Broekema A, Visser B, Brons A, Timmerman A, van Etten-Jamaludin F, et al. Effectiveness of serious games to increase physical activity in children with a chronic disease: systematic review with meta-analysis. J Med Internet Res 2020 Apr 1;22(4):e14549 [FREE Full text] [doi: 10.2196/14549] [Medline: 32234697]

7. Meekes W, Stanmore EK. Motivational determinants of exergame participation for older people in assisted living facilities: mixed-methods study. J Med Internet Res 2017 Jul 6;19(7):e238 [FREE Full text] [doi: 10.2196/jmir.6841] [Medline: 28684385]

8. Tobaigy A, Alshehri MA, Timmons S, Helal OF. The feasibility of using exergames as a rehabilitation tool: the attitudes, awareness, opinions and experiences of physiotherapists, and older people towards exergames. J Phys Ther Sci 2018 Apr;30(4):555-562 [FREE Full text] [doi: 10.1589/jpts.30.555] [Medline: 29706705]

9. Moni MA, Ali AB. HMM Based Hand Gesture Recognition: A Review on Techniques and Approaches. In: 2nd IEEE International Conference on Computer Science and Information Technology. 2009 Presented at: ICCSIT'09; August 8-11, 2009; Beijing, China p. 433-437. [doi: 10.1109/iccsit.2009.5234536]

10. Ibañez R, Soria A, Teyseyre A, Campo M. Easy gesture recognition for Kinect. Adv Eng Softw 2014 Oct;76:171-180. [doi: 10.1016/j.advengsoft.2014.07.005]

11. Antón D, Goñi A, Illarramendi A. Exercise recognition for Kinect-based telerehabilitation. Methods Inf Med 2015;54(2):145-155. [doi: 10.3414/ME13-01-0109] [Medline: 25301322]

12. Raheja J, Minhas M, Prashanth D, Shah T, Chaudhary A. Robust gesture recognition using Kinect: a comparison between DTW and HMM. Optik 2015 Jun;126(11-12):1098-1104. [doi: 10.1016/j.ijleo.2015.02.043]

13. Calin AD. Gesture Recognition on Kinect Time Series Data Using Dynamic Time Warping and Hidden Markov Models. In: 18th International Symposium on Symbolic and Numeric Algorithms for Scientific Computing. 2018 Presented at: SYNASC'18; September 24-27, 2016; Timisoara, Romania. [doi: 10.1109/synasc.2016.049]

14. Calin AD, Pop HF, Boian RF. Improving Movement Analysis in Physical Therapy Systems Based on Kinect Interaction. In: Proceedings of the 31st International BCS Human Computer Interaction Conference. 2017 Presented at: BCS HCI'17; July 3-6, 2017; Sunderland, England. [doi: 10.14236/ewic/hci2017.87] 
15. Hondori HM, Khademi M. A review on technical and clinical impact of Microsoft Kinect on physical therapy and rehabilitation. J Med Eng 2014;2014:846514 [FREE Full text] [doi: 10.1155/2014/846514] [Medline: 27006935]

16. Chang Y, Han W, Tsai Y. A Kinect-based upper limb rehabilitation system to assist people with cerebral palsy. Res Dev Disabil 2013 Nov;34(11):3654-3659. [doi: 10.1016/j.ridd.2013.08.021] [Medline: 24012594]

17. Duarte N, Postolache O, Scharcanski J. KSGphysio - Kinect Serious Game for Physiotherapy. In: International Conference and Exposition on Electrical and Power Engineering. 2014 Presented at: EPE'14; October 16-18, 2014; Iasi, Romania. [doi: 10.1109/icepe.2014.6969981]

18. Cary F, Postolache O, Girao P. Kinect Based System and Serious Game Motivating Approach for Physiotherapy Assessment and Remote Session Monitoring. In: Proceedings of the International Conference on Sensing Technology. 2014 Presented at: ICST'14; October 6-7, 2014; Coventry, UK. [doi: 10.21307/ijssis-2019-131]

19. Su C, Chiang C, Huang J. Kinect-enabled home-based rehabilitation system using dynamic time warping and fuzzy logic. Appl Soft Comput 2014 Sep;22:652-666. [doi: 10.1016/j.asoc.2014.04.020]

20. Ferreira D, Oliveira R, Postolache O. Physical Rehabilitation Based on Kinect Serious Games. In: Eleventh International Conference on Sensing Technology. 2017 Presented at: ICST'17; December 4-6, 2017; Sydney, Australia. [doi: 10.1109/icsenst.2017.8304512]

21. Rybarczyk Y, Kleine Deters J, Cointe C, Esparza D. Smart web-based platform to support physical rehabilitation. Sensors (Basel) 2018 Apr 26;18(5):1334 [FREE Full text] [doi: 10.3390/s18051344] [Medline: 29701690]

22. Just Dance 2018. Wikipedia. 2019. URL: https://en.wikipedia.org/wiki/Just Dance 2018 [accessed 2019-12-03]

23. Palma C, Augusto S, Francisco V. HMM Based Evaluation of Physical Therapy Movements Using Kinect Tracking. In: International Symposium on Visual Computing. 2015 Presented at: ISVC'15; December 14-16, 2015; Las Vegas, NV. [doi: 10.1007/978-3-319-27857-5 16]

24. Kleine Deters J, Rybarczyk Y. Hidden Markov Model approach for the assessment of telerehabilitation exercises. Int J Artif Recogn 2018;16(1):1-19 [FREE Full text]

25. Wei W, Lu Y, Rhoden E, Dey S. User performance evaluation and real-time guidance in cloud-based physical therapy monitoring and guidance system. Multimed Tools Appl 2017 Nov 6;78(7):9051-9081. [doi: 10.1007/s11042-017-5278-5]

26. Richter J, Wiede C, Shinde B, Hirtz G. Approach using Incremental Dynamic Time Warping and Normalised Hierarchical Skeleton Joint Data. In: 6th International Conference on Pattern Recognition Applications and Methods. 2017 Presented at: ICPRAM'17; February 24-26, 2017; Porto, Portugal. [doi: 10.5220/0006108002810288]

27. Vakanski A, Ferguson JM, Lee S. Metrics for performance evaluation of patient exercises during physical therapy. Int J Phys Med Rehabil 2017 Jun;5(3):- [FREE Full text] [doi: 10.4172/2329-9096.1000403] [Medline: 28752104]

28. Palma C, Augusto S, Francisco V. HMM and DTW for evaluation of therapeutical gestures using kinect. arXiv 2016:- epub ahead of print [FREE Full text]

29. Yurtman A, Barshan B. Automated evaluation of physical therapy exercises using multi-template dynamic time warping on wearable sensor signals. Comput Methods Programs Biomed 2014 Nov;117(2):189-207. [doi: 10.1016/j.cmpb.2014.07.003] [Medline: 25168775]

30. Hakim T, Ilan S. A-MAL: Automatic Motion Assessment Learning from Properly Performed Motions in 3D Skeleton Videos. In: International Conference on Computer Vision Workshop. 2019 Presented at: ICCVW'19; October 27-28, 2019; Seoul, Korea. [doi: 10.1109/iccvw.2019.00198]

31. Yu X, Xiong S. A dynamic time warping based algorithm to evaluate kinect-enabled home-based physical rehabilitation exercises for older people. Sensors (Basel) 2019 Jun 28;19(13):2882 [FREE Full text] [doi: 10.3390/s19132882] [Medline: $\underline{31261746]}$

32. Liao Y, Vakanski A, Xian M. A deep learning framework for assessing physical rehabilitation exercises. IEEE Trans Neural Syst Rehabil Eng 2020 Feb;28(2):468-477. [doi: 10.1109/tnsre.2020.2966249]

33. Minsu J, Kim D, Kim Y, Kim J. Automated Dance Motion Evaluation Using Dynamic Time Warping and Laban Movement Analysis. In: International Conference on Consumer Electronics. 2017 Presented at: ICCE'17; January 8-10, 2017; Las Vegas, NV, USA. [doi: 10.1109/icce.2017.7889261]

34. Laraba S, Tilmanne J. Dance performance evaluation using hidden Markov models. Comp Anim Virtual Worlds 2016 May 3;27(3-4):321-329. [doi: 10.1002/cav.1715]

35. Andrei C, Cosmin M, Andrei D, Alina C. Mira. In: Proceedings of the 31st British Computer Society Human Computer Interaction Conference. 2017 Presented at: HCI'17; July 3-6, 2017; Sunderland, UK. [doi: 10.14236/ewic/hci2017.70]

36. Moldovan I, Calin A, Cantea A, Dascălu L, Mihaiu C, Ghircău O, et al. Development of a New Scoring System for Bilateral Upper Limb Function and Performance in Children With Cerebral Palsy Using the Mira Interactive Video Games and the Kinect Sensor. In: Proceedings of the International Conference on Disability, Virtual Reality and Associated Technologies. 2014 Presented at: ICDVRAT'14; September 2, 2014; Gothenburg, Sweden.

37. McGlinchey M, Curtis A, Ritchie R, Jones GD. Feasibility of using MIRA with adult patients presenting with upper limb motor dysfunction post neurological damage. Stud Health Technol Inform 2015;217:696-702. [Medline: 26294550]

38. Czako N, Silaghi C, Vizitiu RS. Rehabilitation of a 7-Year-Old Boy With Brachial Plexus Palsy Using Mira Exergames and Vojta Therapy. In: E-Health and Bioengineering Conference. 2017 Presented at: EHB'17; June 22-24, 2017; Sinaia, Romania. [doi: 10.1109/ehb.2017.7995433] 
39. Moldovan I, Tric L, Ursu R, Podar A, Calin AD, Cantea A, et al. Virtual Rehabilitation Programme Using the MIRA Platform, Kinect and Leap Motion Sensors in an 81 Years Old Patient With Ischemic Stroke. In: E-Health and Bioengineering Conference. 2017 Presented at: EBC'17; June 22-24, 2017; Sinaia, Romania. [doi: 10.1109/ehb.2017.7995427]

40. Unity Real-time Development Platform. URL: https://unity.com [accessed 2020-07-15]

41. Sakoe H, Chiba S. Dynamic programming algorithm optimization for spoken word recognition. IEEE Trans Acoust Speech Signal Process 1978 Feb;26(1):43-49. [doi: 10.1109/tassp.1978.1163055]

42. Hu M, Chen C, Cheng W, Chang C, Lai J, Wu J. Real-time human movement retrieval and assessment with kinect sensor. IEEE Trans Cybern 2015 Apr;45(4):742-753. [doi: 10.1109/tcyb.2014.2335540]

43. Rabiner LR. A tutorial on hidden Markov models and selected applications in speech recognition. Proc IEEE 1989;77(2):257-286. [doi: 10.1109/5.18626]

44. Chen C, Roozbeh J, Nasser K. UTD-MHAD: A Multimodal Dataset for Human Action Recognition Utilizing a Depth Camera and a Wearable Inertial Sensor. In: International Conference on Image Processing. 2015 Presented at: ICIP'15; September 27-30, 2015; Quebec City, QC, Canada. [doi: 10.1109/icip.2015.7350781]
Abbreviations
3D: 3-dimensional
DTW: dynamic time warping
HMM: hidden Markov model
KEHR: Kinect-enabled home-based rehabilitation system
MIRA: Medical Interactive Recovery Assistant

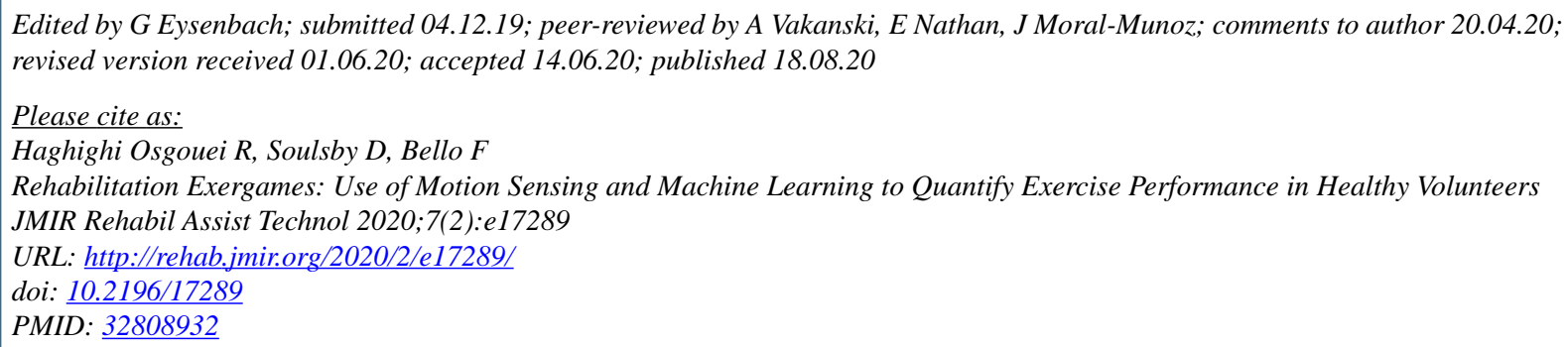

CReza Haghighi Osgouei, David Soulsby, Fernando Bello. Originally published in JMIR Rehabilitation and Assistive Technology (http://rehab.jmir.org), 18.08.2020. This is an open-access article distributed under the terms of the Creative Commons Attribution License (https://creativecommons.org/licenses/by/4.0/), which permits unrestricted use, distribution, and reproduction in any medium, provided the original work, first published in JMIR Rehabilitation and Assistive Technology, is properly cited. The complete bibliographic information, a link to the original publication on http://rehab.jmir.org/, as well as this copyright and license information must be included. 